\title{
Ungaretti e il sentimento della guerra
}

\author{
Stefano VERDINO \\ Università degli Studi di Genova ${ }^{1}$ \\ stefano.verdino@unige.it
}

Recibido: $27 / 03 / 2015$

Aceptado: 30/04/2015

\section{RIASSUNTO}

Attraverso l'analisi comparativa di lettere a diversi interlocutori (Papini, Marone, Soffici, Puccini, Prezzolini) si delineano i diversi sentimenti della guerra del fante Ungaretti dal 1915 al '18. Negli anni permane una fede nel senso di quel sacrificio, che ha però sempre più i connotati del massacro. Nelle lettere affiora un circuito tra i dati della solitudine e della sofferenza con l'esigenza della fraternità e della gentilezza, quali fondamentali antidoti per resistere.

Parole chiave: lettere, solitudine, sofferenza, fraternità, gentile.

\section{Ungaretti and the sentiment of the war}

\begin{abstract}
The comparative analysis of the letters sent to some interlocutors (Papini, Marone, Soffici, Puccini, Prezzolini) allows to define the changes in the attitude of the soldier Ungaretti towards the war from 1915 until 1918. The letters show up how the sense of fraternity and kindness can oppose the solitude and the pain becoming the main resources to endure the war.
\end{abstract}

Key words: letters, solitude, pain, fraternity, fine.

«Carissimo Papini, ci siamo», così scrive il fante Ungaretti appena giunto in «zona di guerra» il 5 dicembre '15, su una cartolina postale in franchigia (Ungaretti 1988: 17). Dopo due settimane e poco più di fronte Veglia (cima quattro 23 dicembre 1915) è la prima poesia che ci "butta" - per continuare la brutale espressione del testo - nella guerra. Un testo epocale, che da quasi un secolo sigla

${ }^{1}$ Dipartimento di Italianistica, Antichistica, Romanistica, Arti e Spettacolo, via Balbi 2, I-16126 - Genova. 
l'esperienza poetica italiana di trincea in tutte le antologie ${ }^{2}$, repertori, siti; un testo che - a differenza dell'usuale inquieto variantismo dell'autore - non ha conosciuto mutamenti (tranne la lieve dislocazione finale) ${ }^{3}$.

Il battesimo - quasi natalizio - di Veglia ci offre gli elementi cruciali del sentimento della guerra ${ }^{4}$, sostanzialmente mai revocati dal poeta, che possiamo così rubricare nel loro apparire. Il tempo lungo («Una intera nottata»), la violenza subita («buttato»), la stretta contiguità con i commilitoni morti («vicino a un compagno»), le gamme dell'orrore fisico dei cadaveri («massacrato», «bocca digrignata», «congestione delle sue mani») «volte» alla maestà della natura («plenilunio») e penetranti il proprio intimo («penetrata nel mio silenzio»); in questo circuito di morte-natura-io scatta la reazione di scrivere «lettere piene d'amore», con il conseguente commento e la dichiarazione di essere «tanto attaccato alla vita», che in simmetria (e rima) all'iniziale «buttato» corregge l'esito del testo, dalla morte alla vita, che sarà come noto il leitmotiv di tutta l'Allegria.

Ma il mio interesse ora non è quello di ripercorrere la poesia di Ungaretti, quanto mai criticamente disossata da tempo, piuttosto osservare i dati rilevati della guerra, nel circuito tra versi e le molte lettere dal fronte, secondo la sua veritiera affermazione di Veglia. Come sappiamo Ungaretti poeta non insiste più di tanto sull'orrore, "l'espressionismo" di Veglia è anche un unicum, rispetto al martellare di un Rebora. Ungaretti è certo il poeta italiano che più di tutti ha vissuto al fronte e ha vissuto la guerra, in tutte le sue fasi e i suoi volti, tra assalti e pause, molte pause: «Questa guerra non è eroismo è pazienza. Ho in orrore la pazienza!» scrive a Papini (timbro 6-4-1916; Ungaretti 1988: 23). La guerra è anche «questo vuoto», come si legge in un appunto di versi inviato a Papini nell'aprile del '16 (Ungaretti 2009: 458), ed anche il «deserto» ${ }^{6}$; vi si può imputare la «nevrastenia», di cui Ungaretti

${ }^{2}$ Ma curiosamente due ungarettiani della prim'ora come Papini e De Robertis non la accolsero nelle loro crestomazie; la poesia si impose dai Lirici nuovi di Anceschi (1943).

${ }^{3}$ In Il Porto sepolto (Ungaretti 1981a: 44): «Non sono mai stato / tanto attaccato / alla vita»; nell'Allegria definitiva: «Non sono mai stato / tanto / attaccato alla vita» (Ungaretti 2009: 63).

${ }^{4} \mathrm{Su}$ Ungaretti e la prima guerra mondiale vedi le pagine di Cortellessa (1998) che rubrica Ungaretti nelle sezioni «La guerra-comunione», «La guerra-percezione» e «La guerra-lutto», rilevando sia le nuove modalità percettive che la guerra impone, sia il nuovo statuto dell'io. Altre osservazioni sulla guerra per Ungaretti in Cortellessa (2000), BultriniFabi (2007) e Thompson (2009).

5 Vedi Saccone (2012: 65); a questo libro si rimanda per la precedente bibliografia (Saccone 2012: 273-85); vedi inoltre ancora sul tema della grande guerra De Michelis (2014) e De Liso (2015).

${ }^{6}$ A Papini (timbro 31-12-1915): «Sapesse in che deserto mi trovo. Ho ricevuto da Parigi 'Le Journal des Ecrivains'. È la sola carta stampata che mi sia pervenuta da settimane. A darmi notizie di morti! Ho fatto le mie giornate di trincea sulla cresta d'un monte, affogato nel fango. Ma questo sarebbe nulla. È altro che mi deprime. Tornato in Italia le scriverò. Per ora, tranne quando trascino il mio corpo riottoso a combattere, sono un decaduto» (Ungaretti 1988: 17-18). 
dice d'aver sempre sofferto, ovvero in termini poetici, la sublime analogia della «corolla di tenebre», cui somiglia la propria vita. Scrive a Papini, il 29 luglio 1916:

Dicevo: ritrovarmi primitivo è stata anche una sorpresa, sulle prime, e anche ora, a volte: ma poi il neurasténico prevale, e allora è una crisi.

S'era arrivati a capire che al mondo, in mano agli uomini, non c'è che una forza: distrarsi. Riverrà quel tempo, anche per me? L'avrei pagato, in tanto tempo attanagliato alla morte; - e sai che allora non s'è distratti - .

No, nessun brrrrr; ché poi, se madama morte arriva, è uno stiracchiamento di membra (diciamo, per esser sinceri: colle povere membra violentate e straziate - Dio, chiudiamo gli occhi! - ; la buona morte classica anche quella è una nostalgia ma morire, con coraggio, in un assalto, dev'essere un supremo abbandono alla vita, un'estasi totale -) uno stiracchiamento di membra, e un riposo definitivo, e amen; ma il permanente accorgersi che tutto è così perfettamente inutile... Era una gran / nobiltà aver provato che lasciarci vivere, senz'altro, è la verità; era una gran raffinatezza! (Ungaretti 1988: 65)

Ma nel vuoto deserto si possono accampare i miracoli e le parole che essi suggeriscono, dalla fulminea intensità di Mattina al racconto di I fiumi, che ben vanno contestualizzati come tangibili miracoli per chi vive nell'angusto della trincea e privo di un bagno.

Il poeta, avvezzo al deserto, privilegia miraggi e miracoli incentrati su di sè («Ungaretti / uomo di pena / ti basta un'illusione per farti coraggio», Ungaretti 2009: 64) ed è parco e anche refrattario a testimoniare la guerra, sia come comune impresa (alla Soffici), sia come indicibile orrore (alla Rebora), sia come distanziamento dal proprio io, con buone iniezioni d'ironia (alla Sbarbaro). In effetti le tre sezioni centrali dell'Allegria, che racchiudono ciascuno un anno di guerra (1915-16, Il porto sepolto; 1917, Naufragi, 1918, Girovago), manifestano un progressivo attutimento dalla specifica vicenda militare: in Porto sepolto sono cospicui i puntuali riferimenti, oltre che in Veglia si hanno in Peso ( Quel contadino / si affida alla medaglia / di Sant'Antonio / e va leggero», Ungaretti 2009: 72), nel notissimo Fratelli, nei due testi sulle trincee, quella in combattimento di In dormiveglia ( ${ }^{7}$ L'aria è crivellata / come una trina / dalle schioppettate / degli uomini / ritratti nelle trincee / come le lumache nel loro guscio», Ungaretti 2009: 80) e quella in allerta di Pellegrinaggio ( «In agguato / in queste budella / di macerie / ho strascicato / la mia carcassa / usata dal fango», Ungaretti 2009: 84), nel «paese più straziato» di $S$. Martino del Carso, fino alla chiusa ideologica del «poeta» e «soldato» di Italia. L'anno dopo - il tragico '17 - dolente per la forte nevrastenia (non a caso racchiude una sezione che si intitola Naufragi) è tutta in punti di fuga tra ricordi, sogni, miracoli; solo Vanità si ricorda delle «macerie», subito travolte dal «limpido stupore dell'immensità»; infine Girovago (che allude alle diverse dislocazioni di Ungaretti nell'ultima fase del conflitto) ha solo nel titolo Soldati uno specifico riferimento al conflitto in corso. Quindi progressivamente sempre meno. Il

\footnotetext{
${ }^{7}$ Nel Porto sepolto 1916 con il più testimoniale titolo Immagini di guerra.
} 
silenzio delle poesie è a suo modo eloquente, di una condizione che ha - in questi testi - sempre luogo e tempo (le precise datazioni), ma non le parole, le quali tendono ad accamparsi "altrove", con un effetto cortocircuito o paradosso certo assai significativo.

Se il poeta di guerra è l'Ungaretti del primo Porto sepolto (che non a caso nella bibliografia critica - tende ad avere vita autonoma, rispetto all'intruppamento autoriale nell'Allegria), diverso è il discorso per le molte lettere dal fronte scritte e necessarie alla propria stessa esistenza. Della varia corrispondenza edita emerge la figura di Papini, interlocutore privilegiato: alle istanze "rigeneratrici" della guerra di Papini aderisce il soldato Ungaretti, ma con il tono sgomento di chi in trincea ne vede l'orrido costo:

Mio Dio! purché veramente n'esca più grande l'Italia; più intensamente viva; risvegliata in tutto il suo vigore; nella sua piena civiltà; ché se in una volta turbinassero al cielo $i$ suoi colori infiniti, sarebbe un sole questa nostra patria! (timbro 11-7-1916; Ungaretti 1988: 59)

Esattamente un anno dopo, nel luglio '17, sempre con Papini, la guerra si rivela «tremenda sofferenza», in un'analisi molto lucida e complessa del diffuso sentimento del «bene [...] perduto» da una parte e dall'altra di un sentimento «meraviglioso» della vita, che però va sfuggendo con l'incalzare della morte, che stimola sentimenti assai meno baldanzosi di quelli della lettera del 29 luglio '16 a Papini sopra ricordata:

Sono soverchiato dalla guerra, da questa tremenda sofferenza. Posso anche cantare; è un modo infernale di piangere che la natura ha dato in dono alla gente battezzata dal sole; che è la gente più malinconica; di cui ogni momento è la nostalgia del momento passato; la nostalgia ch'è il fiele della malinconia; si passa il tempo a sospirare sdegnati qualcosa d'irrimediabilmente perduto, e che poc'anzi c'era; e poc'anzi era la stessa cosa, con lo stesso desiderio inappagabile; un'irrisione. La vita è una cosa così meravigliosa per noi, che non ci si può rassegnare alla morte; non si può sentire il passato senza una desolazione straziante; e da tre anni s'è abbattuti sotto il calcagno della morte che ti mostra i denti «necessari» della «disciplina». Da tre anni gli uomini più cari ci spariscono; in Francia ci mancano già tanti; chi c'è rimasto? e da noi, s'è allontanato Renato [Ettore] Serra; e noi che non abbiamo fatto nulla, che non siamo morti, abbiamo perduto la fede; la vita non si vede più che nella morte; e siamo puniti di non essere morti. (Ungaretti 1988: 126-127)

Ma la «fede» che qui si dà perduta pochi giorni dopo si riaffaccia nei confronti di «questo paese ch'è "nostro"» e si rivendica il proprio essere «uniforme» (quasi «docile fibra» di un popolo), «ma di molto scetticismo e rancore s'è acquistato» mentre con piena lucidità e genialità di immagine si rimarca che «ogni giorno è abbattuta quest'insuperabile organizzazione umana ch'è un nostro contadino, a migliaia» (timbro 18-7-1917; Ungaretti 1988: 127). E pochi giorni dopo (timbro 78-1917) - e dopo la lettera dell'«inutile strage» (1 agosto 1917) del Papa - la stessa 
parola «strage» si affaccia da un'altra lettera a Papini in cui accanto alla consolidata rivendicazione del «sacrificio» si imputa ai tedeschi la responsabilità della «strage», nonché lo snaturamento del costume dell'italiano non avvezzo alla «pazienza»:

Come agiatezza non potrei chiedere né desiderare condizioni migliori di quelle mie attuali, facendo il militare; purché la duri.

Come morale; dura da un po' troppo; ed è necessario, malgrado tutto - restar tenaci; dopo tanto sacrifizio; chi avrebbe il coraggio, - chi reggerebbe al suo volto d'uomo -, di tornare indietro per nulla; mille volte meglio morire tutti. Forse i tedeschi fanno anche loro questo ragionamento. Ma perché hanno mosso questa strage? Ma perché sono così grossolani e perfidi? Gesti da orso e cervello da volpe; e per cuore hanno una vescica di birra; ma sanno star imbrancati come una macchina; hanno i nervi d'acciaio. Li odio, li ho in orrore, sopratutto perché mi hanno costretto alla pazienza; un italiano paziente; per colpa loro si deve sopportare, è necessario sopportare, anche quest'altro - il principale di questa guerra - contro-natura; fare come loro. (Ungaretti 1988: 138)

Anche dopo Caporetto l'obbedienza di Ungaretti non viene meno («bisogna star qui, vivere qui, patire qui, e anche questo è necessario»), ma accanto all'infittito logoramento («quante concitate giornate sotto quel grigio sudicio che copre il cielo, nel mio tempo, da 3 anni») merita dar conto di un crescente sentimento di «solitudine» e di distanza dal paese che ha vissuto tre anni diversamente, come scrive a Soffici (timbro 2-2-1918):

Una delle cose più terribili per uno di noi in questa guerra è l'isolamento. In fin dei conti la vita ha continuato il suo corso. In paese (in Francia nostra come in Italia nostra) da una parte e al fronte dall'altra; quelli del fronte, - da noi credo eccessivamente più che in Francia -, non hanno avuto da parte di quegli altri quella solidarietà che la loro condizione imponeva, tranne rare eccezioni. (Ungaretti 1981b: 10-11)

Se queste in sintesi sono le idee sulla guerra corrente del fante Ungaretti, registriamo sì sofferenza e angoscia, ma mai protesta; è una guerra come «azzardo» tra vita e morte ad imporsi, cui egli «aggioga» se stesso e la propria ragione di vita:

Caro Papini, ti scrivo per rendermi conto di me stesso. Quanto sacrifizio ci vorrà ancora per vincere? Si misura colla mia vita? Posta così la mia vita è insignificante. Ma sono implicato; sono aggiogato alla guerra. La guerra, dopo due anni - per me Francia e Italia è tutt'uno - ci costa troppo, per fermarci senza frutto. E mi attacco alla mia vita con disperazione; non posso stare in quest'azzardo di cedere tutto per nulla, senza sgomento. Eccomi, Papini. Perché la morte significa annullarmi, per me, ho paura di morire, e forse dovrò presto morire. (timbro 23-3-1916; Ungaretti 1988: 19)

Come in I fiumi e in altri versi, le lettere informano in vario modo della prigionia di un corpo giovane incatenato alla trincea: 
sono tutto acceso; siamo tutti accesi; quest'estate birbona; e senza rinfreschi di nessuna specie; cré nom de Dieu! T'assicuro che il povero corpo urla; questa povera belva incatenata di grigioverde! (a Papini, timbro 11-7-1916; Ungaretti 1988: 59)

Se pesa la sfasatura dei propri desideri con la maestà della natura ${ }^{8}$, non mancano notizie di ragni, topi e pidocchi, paradossalmente quasi rinfrancanti ${ }^{9}$ e l'autoritratto di sé che discende dai versi e si ritrova nelle lettere è quello di un uomo sorridente quanto impietrito, come si legge in una lettera all'amico egiziano Jean-Léon Thuile (timbro 4-10-1917):

Sono due anni a momenti che vivo in questo delirio e non si può descrivere quel che ho patito, ogni specie di sacrificio, sono stato il povero corpo e la povera anima buttati violentemente. Sono rimasto col sorriso sulle labbra giacché la mia sorte era quella dell'umanità travolta. Mi s'è scavata soltanto ai margini della bocca qualche ruga di pietra. [...] Ma oggi le ulcere profonde sono di più pungenti. Sono incancrenite. (Livi 1988: 78-79)

Anche l'esperienza di una ferita superficiale nel '18 è vissuta come prova superata e diventa viatico di un progetto di canto corale, di cui scrive in modo identico a Papini e a Prezzolini (timbro 18-7-1918):

Mio caro Papini, vivo per miracolo. Una scheggia mi ha rotto il fucile e mi ha graffiato la tempia. Ma la morte non mi vuole. Tornando dal combattimento dove gli italiani sono stati degni «si resiste o si muore», ero in uno stato tale di esaurimento, per le lunghe fatiche e privazioni e emozioni, che avevo perdite continue e ogni tanto cadevo in deliquio. Senza il soccorso del mio colonnello che fece fermare un camion perché mi caricasse, sarei finito sulla strada. Scriverò il canto di queste atroci e miracolose giornate. (Ungaretti 1988: 214; Ungaretti 2000: 55)

E siamo al punto del rapporto tra il fante Ungaretti e i suoi compagni, i soldati, un rapporto che sappiamo dai versi di cordiale sintonia e che nelle lettere si ritrova

${ }^{8}$ A Papini, luglio 1917: «mi raggiunge lo spasimo delle cicale ubbriache, e il rotolìo incessante dei veicoli; e il passo degli uomini che scendono e salgono, incessante; e questo peso della strada, e questo peso del sole, che sono cose da godersi a proprio talento; mentre così imbrancati, ci mettono soltanto nel sangue un pimento di desideri che bisognerà veder corrompersi strangolati in noi» (Ungaretti 1988: 125).

9 A Papini (timbro 8-9-1916): «C'è da registrare una novità; ora la bestia del giorno è il ragno; ogni tanto ci sentiamo addosso la delicatezza delle sue gambine; e per farne preda, per un attimo non vediamo più il panorama orrendo» (Ungaretti 1988: 71); a Carrà, 26-21917: «Mi sono coricato sui sassi fangosi, e topi, come gatti, mi passavano addosso come fossi stato casa loro, e i pidocchi graziose bestioline, un po' torbide ma tenaci come tedeschi, mi mangiavano allegramente; ma la mia fantasia non aveva soste che per contemplare se stessa a bearmi d'esser sempre me stesso, così adagiato così irresidualmente transfuso nella vita» (Piccioni 1980: 95-96). 
disteso in varie occasioni: si legge la mitizzazione, eroica, della presa di un villaggio:

Caro Papini, oggi è Pasqua, una domenica banale, e piove. Ma tu avessi visto, stamani, i nostri soldati, i miei compagni, come fissavano il loro mondo custodito, con che smarrimento si ritrovavano per le strade di questo villaggio «redento». Cari miei compagni. Sfilavano ieri snelli alla cadenza dei loro passi risoluti, amabili. E hanno guardato in viso la morte, senza sapere perché, e hanno ripreso il monte «aspro», i più ingegnosi i più mansueti i più impetuosi i più generosi - non sanno perché - ricostruttori di questa terra, italiani. (timbro 24-4-1916; Ungaretti 1988: 26-27) ${ }^{10}$

Dominano $\mathrm{i}$ flash di comune allegria, dalle marce alle ascese ${ }^{11}$, ai racconti contadini $^{12}$, con una costante mitologizzazione identitaria ${ }^{13}$, individuata nell'improvvisazione, segno di vitalità:

Ieri ho assistito qui - in questo paese pieno di mosche - a uno spettacolo grazioso: dei soldati a coppie - uno a cavallo dell'altro - giocavano al pallone fra matte risate; non erano bimbi, ma uomini maturi - ; con il loro elmo sul capo; - a così breve distanza dalla guerra, già tanto lontani - ; l'italiano mi riempirà sempre di meraviglia; quando un uomo è così carico di vita, sara sempre il re della creazione. (a Papini, timbro 22-7-1917; Ungaretti 1988: 130, identica a Puccini, stessa data, Ungaretti 2015: 48) ${ }^{14}$

10 Dal San Michele conquistato scrive a Papini (timbro 10-8-1916): «Ho visto cose meravigliose: il miracolo: i feriti non avevano dolori: gli altri non potevano essere frenati: era un grido di una passione infinita: "Si vede il mare, si vede il mare": lo spazio finalmente, Papini: fuori di pazienza, ci siamo arrivati» (Ungaretti 1988: 66).

${ }^{11}$ A Papini (timbro 29-6-1916): «L'altra notte mi sono fatto una marcia di una decina di chilometri, sotto una pioggia torrenziale, mi sono sfogato a cantare cogli altri soldati; non mi ricordavo più di me; era una felicità» (Ungaretti 1988: 48). «Mio caro Papini, siamo risaliti. Un soldato portava in braccio la Checca (la gazza), un altro un gattino vispo trovato in una delle case dove si accantonava; questi italiani sono proprio belli; vanno così ad affrontare la morte, spensierati e dolci; com'è confortante: come non si dovrebbe vincere?» (17 luglio 1916; Ungaretti $1988: 63$ ).

12 A Prezzolini, ante 7-2-1917: «Mi faccio raccontare da un soldato pieno di arguzia popolana la storia di Giovannin bel grosso; e sto estatico come gli altri soldati ad ascoltarlo in giro al fuoco; mi sento tanto popolo, pieno d'infanzia, ch'è stupore, entusiasmo, poesia e tutte le altre belle cose; e vado in branda con qualche sogno, caldo e ristorato» (Ungaretti 2000: 41).

${ }^{13}$ A Papini (timbro 11-9-1917): «Il popolo nostro è un popolo di fantastici; ha più d'ogni altro, anzi questo è il suo carattere, il potere di transmutare la realtà in ebrezza» (Ungaretti 1988: 145).

${ }^{14}$ Assai raro è trovare cenno a disagi fisici (ma certo la censura avrebbe operato), per cui spicca questo miniracconto a Papini del 1918: «Ho viaggiato con l'emicrania e una profonda amarezza. So che ho dovuto rimanere quasi sempre in piedi e pigiato come una salacca, in un carro bestiame "cavalli otto uomini quaranta" come lo chiamano i militari, i miei compagni, che mangiavano e bevevano; avevano tutti dei fagottoni di provviste malgrado la 


\author{
Altrove è l'uniforme a dare piena identità a quelli che altrimenti sarebbero \\ «poveri cristi»» ${ }^{15}$
}

Mio caro Papini, sono ancora con te stasera; una calma sera; non un'ombra sul cielo, e sulla terra il solito vagare; quando gli uomini non sono più imbrancati e trasportati dal freno della guerra con quella loro pesante monotona cadenza collettiva nelle membra, non più loro - della società! - , in questo paesello, a quest'ora si vede bene che sono poveri cristi vaganti quaggiù chissà perché. (28 febbraio 1917; Ungaretti 1988: 106)

Ungaretti osserva anche i propri compagni mentre come lui scrivono «lettere piene d'amore», ad antidoto ed osserva le difficoltà di “dire la guerra", anche se può esserci - anche in questo caso - un miracolo, magari nel rango di una smorfia:

Caro Papini, i miei compagni scrivono, accoccolati nelle tane, in mezzo a un fracasso ch'è ormai, per noi, una monotonia.

Per ammazzare il tempo fermo quest'altro foglietto. In sé le lettere dei soldati sono la cosa meno spontanea di questa terra. In generale, perché c'è chi indovina delle espressioni efficaci come questa: «i 305 passano sulle nostre teste come treni». - Ma le pappolate di un Barzini o di un Civinini, ridotte così a smorfie, acquistano un interesse, riescono corrosive. (timbro 24-3-1916; Ungaretti 1988: 20)

carestia, e non si lamentavano. Un tale, un sergente parlava continuamente di un "porcilento" che in una camerata di quattro metri, dove doveva dormire con 25 compagni, sulla paglia piena di pidocchi "anche il naso mi mangiavano i pedocci", di un "porcilento" che non aveva riguardo di pisciarci in un cantone: "ma una volta l'ho preso nel buio, e gli ho pestato il muso a quel "porcilento"[...] Non so fare uno sgarbo a un uomo, ma un porcilento è un porcilento". Insomma non ho capito altro attraverso al fumo dei sigari, delle pipe, all'odore del vino e del mangiarne e dell'uomo, a tutto quel tanfo forte, compresa la mia amarezza, che il "porcilento" che ritornava come un battito d'orologio, in mezzo a un grande silenzio» (Ungaretti 1988: 192-3).

${ }^{15}$ Discorso certo non razziale in Ungaretti, che lo riferisce anche a se stesso, scrivendone a Puccini (timbro 11-7-1917): «Ma ho bisogno di tornare al mio reggimento. Ne ho condiviso le sorti per 16 mesi. Vi ho patito, e vi ho trovato tanto affetto, dal colonnello al più umile soldato; vi ero idolatrato. Ti chiedo questo poco. Chiedo da una presidiarla di riandare a un reggimento combattente, al mio $19^{\circ}$. Mi pare che questo poco si possa ottenere. Ma presto» (Ungaretti 2015: 38). A Soffici (timbro 8-2-1918): «Cari buoni soldati; non ho mai avuto uno sgarbo da parte loro, mai, ma prove continue d'un'umanità, che è veramente la sola che mi rinfranchi nell'ore più tristi; se arrivo tardi dove dormiamo fanno a gara per farmi posto; se in un'osteria li colgo a lamentarsi della guerra, mi basta guardarli un po' negli occhi per sentirli riprender coscienza, se lo zaino mi dà fatica, me lo tolgono per forza di dosso, e mi vogliono portare il fucile, e mi offrono il braccio; no: i soldati non possono che voler bene a quest'uomo e per lui non avranno mai mai un movimento d'invidia» (Ungaretti 1981b : 13-14). 
La ricerca di un "paese innocente» fa sì che in guerra Ungaretti cerchi un deciso antidoto alla brutalità ed ecco allora il ricorrente campo semantico del «gentile», a siglare una somma di gesti direi (più che valori) umani. Tutti abbiamo in mente il «gentile Ettore Serra», ma nell'epistolario la frequenza semantica è assai rilevante e va da analoghi gesti concreti di rimozione o attutimento della barbarie ${ }^{16}$ a un investimento decisamente più ideologico: «Caro Papini, sono in ansia, e lo sarete voialtri, in questi giorni. Non dovrei pensare ad altro che al mio paese che si batte per "la gentilezza"» (timbro 26-5-1916; Ungaretti 1988: 39).

Non a caso è sempre in nome della «gentilezza» che Ungaretti a più riprese ${ }^{17}$ reagisce dopo la disfatta di Caporetto, nel novembre 17, con un evidente investimento nazionalistico:

Mio Papini, avrai forse ricevuto la cartolina che in tutta fretta ho potuto mandarti appena incontrata una buca postale, la prima dal giorno triste. Non ti posso dire nulla. Si soffre, e malgrado tutto si spera ancora. Grida agli italiani di aver forza d'animo, di resistere: coraggio, Papini; grida con tutta la tua forza al nostro popolo di aver coraggio; se la gentilezza deve scomparire dalla terra, la cederemo a caro prezzo: fino all'ultimo uomo siamo pronti. (Ungaretti 1988:159)

E la parola guarda caso torna nel primo articolo noto di Ungaretti Zona di guerra (vivendo con il popolo) (ne Il Tempo, 4 gennaio 1918) come evidente intenzionalità tonificante e discriminante tra «gentilezza» latina e «formidabili guerrieri», ma implicitamente barbari, tedeschi:

Chi parla oggi di pace è un delinquente. Oggi bisogna resistere; o uccidere la gentilezza. I tedeschi sono dei formidabili guerrieri; peggio, sono dei sicari. Hanno ucciso la patria di Dostoievski; tentano d'assassinare la Francia e d'assassinarci. Diffidate.

Abbiamo in orrore la pazienza; ma saremo pazienti come loro, più di loro. Più dei loro cannoni c'è da temere il loro subdolo intrigante inquinamento. Chi ha responsabilità sulle spalle se ne ricordi, e si ricordi di prepararci presto un piano di vittoria. L'Italia vuol essere più grande, non di territorio, ch'è secondario, ma d'anima, che se lo merita; non vuole essere destituita alla sorte d'una tribù di negri; vuole essere elevata ai fasti che in duemil'anni, senza riposo, traccia nella storia della gentilezza. (Ungaretti 1974: 8-9) ${ }^{18}$

${ }^{16}$ A Papini (timbro 24-3-1916): «allo svolto di una solita giornata, in un canto di rupi, c'è composto un tumulo di fango, e c'è sul fulvo, discreto nell'ombra della sera, una crocina pallida, e s'intrecciano a rami di pino, a mosaico queste parole gentili: "A tre fratelli del 19"» (Ungaretti 1988: 20).

17 A Papini (timbro 19-12-1917): «Ho intenzione di raccontare la storia del vecchio 19 impersonata in una gentilezza, svanita nel silenzio, laggiù; la figura spendente di umanità del capitano Cremona, laggiù nel silenzio di questo mio cuore peso come una pietra» (Ungaretti 1988: 166).

${ }^{18}$ Come per Soffici e altri 'propagandisti' per Ungaretti le colpe non sono dell'esercito, ma dei pacifisti disfattisti: "La ritirata ci ha ucciso il canto. Quando ci scoppierà questo pianto impietrato? I soldati d'Italia non chiedono che d'esser condotti alla vittoria. Per tre 
Di Caporetto Ungaretti accenna più riprese a Papini, ma un racconto puntuale lo si legge in una missiva a Puccini del novembre '17:

dal giorno triste non ho avuto né forza né tempo di scrivere, e neppure di pensare. Ho seguito dal Nad Logem la mia compagnia che cogli ultimi reparti di copertura ha abbandonato quelle posizioni dove ero arrivato al tempo della conquista con il mio 19 circonfuso di gloria. Ho seguito il pellegrinaggio, stordito, per il Vallone ${ }^{5}$ per il San Michele per Sdraussina lungo i cimiteri dove si lasciavano tanti morti che m'erano stati cari in vita, che avevo visto partire schiantati in piena speranza increduli della morte, sebbene docili, poveri compagni lontani. Stordito d'essere ancora, sulla terra, un uomo che sentiva il peso del suo corpo fragile, l'inutilità del suo peso avvilito. Mio Dio che cosa atroce e ossessionante portare così la propria vita viva, sebbene tanto stancata e logorata, quando tutto ci sembra morto; tutto allontanato e noi rimasti non in una Tebaide, ma in uno smarrimento senza senso. Puccini mio, non ho sofferto; sarebbe stata una forza; l'uomo ha la necessità di soffrire come ha quella di respirare l'aria; mi sono sentito senza cuore e senza pensiero, eppure vivo; ma buttato via come una pietra da una violenza bruta. Oggi spero, oggi mi rinasce la speranza, oggi mi rigermoglia la sofferenza, oggi mi sento ancora buono nel sole che scioglie la brina e indiamanta l'erba in questa pianura veneta. Viene la Francia da noi, ho letto; e vorrei chiederti, con la sicurezza di rifare una domanda vana: potrei essere utile? Dopo tre anni di zona di guerra, un soldato può chiedere di essere utilizzato nel punto più pericoloso, ma secondo le sue attitudini? (Ungaretti 2015: 55)

La ritirata ha interrotto il «canto» degli italiani, convertito in «pianto impietrato», ma ciò non deprime Ungaretti che in privato continua a canticchiare ${ }^{19}$

anni hanno atteso con pazienza, che non è nella loro indole, il piano di grande stile degli alleati. Non è venuto. E venuta la ritirata. Nessuno saprà mai perché. Ma vi giuro che il popolo italiano non ha tradito. Ci sono traditori. Sì; oggi chi parla di pace» (Ungaretti 1974: 8). Raccomandazione agli alti gradi di motivare i soldati come scrive a Papini (timbro 1511-1917): «Del nostro soldato si può ancora fare quel che si vuole; una grandezza insuperabile o un'estrema abiezione: un po' di psicologia, un po' di psicologia. Spero, spero spero» (Ungaretti 1988: 160). E successivamente (timbro 9-1-1918): «Ancora in Italia non si rendono conto del significato preciso della ritirata sul morale del soldato; è stato questo: come di qualcuno che avesse un figlioletto malato, e non si rendesse conto della gravità del male; e d'un tratto se n'accorga e perda la testa per l'urgenza e la difficoltà di procurare il soccorso necessario; questo; hai capito? Siamo dunque diversi oggi, siamo veramente disposti a durare anche cent'anni la guerra, a morir tutti, pur di non esser vinti» (Ungaretti 1988: 176).

${ }^{19}$ Vedi i versi, datati 11 giugno 1918, inviati a Papini (e con varianti a Soffici, timbro 12-6-1918; Ungaretti 1981b : 28): «le giornate si affondano nelle notti; i giorni si levano dalle nottate in un coro di mitragliatrici e d'usignoli; pare la delicata la voce intima della brutale; fratelli miei topi che guaite in ruzzo amoroso e mi passate sulla tempia stanca per forzarmi a un ricordo di ribrezzo.

tutto si ferma in un silenzio d'abisso. 
ed anche - con Soffici ( timbro 2-2-1918) - a vaticinare oleografiche ed angeliche riscosse:

All'epoca in cui non c'era ancora morto il canto, cantavano:

"il dicennove e venti

han preso il San Michele

e sulle cartoline

han messo i bersaglieri..."

Quando ci scoppierà questo pianto impietrato in un canto luminoso di vittoria? I tuoi cannoni, Italia, metteranno nella notte, una tempesta di soli: e nel convulso delle mitragliatrici appariranno i tuoi fanti come i cori angelici di Natale. Natale di Gesù. Giornata di agnelli inleoniti, per farti un carnato di bellezza immortale, Italia. (Ungaretti 1981b: 5)

Se questa è la campionatura di un sentimento della guerra in diretta, merita una postilla su come - nei cinquant'anni successivi - Ungaretti sentiva quell'evento. Trent'anni dopo, negli anni per lui difficili dell'immediato secondo dopoguerra, riflettendo sul proprio destino di poeta, non potrà che fare riferimento alla guerra come proprio essenziale «riconoscimento» (lo sappiamo da I fiumi), a parte una comprensibile autoapologia in quei frangenti, la guerra non è più il «sacrificio» delle lettere del '15-18, ma si conferma e radica come «sofferenza», che nella sua riduzione umana manifesta un «primitivismo», foriero di umana solidarietà in Indefinibile ispirazione (scritto tra 1947 e '55):

Fu durante la guerra - ed era il solo insegnamento valido, umano, che la guerra potesse dare, era l'insegnamento che massimamente contraddiceva a quello che se ne aspettava, che ne aspettavano gli utopisti d'ogni sorte - ma fu durante la guerra, fu la vita mescolata all'enorme sofferenza della guerra, fu quel primitivismo: sentimento immediato e senza veli; spavento della natura e cordialità rifatta istintiva dalla natura, spontanea e inquieta immedesimazione nell'essenza cosmica delle cose; - fu quanto d'ogni soldato alle prese con la cecità delle cose, con il caos e con la morte, faceva un essere che in un lampo si ricapitolava dalle origini, stretto a risollevarsi nella solitudine e nella fragilità della sorte umana; faceva un essere sconvolto a provare per i suoi simili uno sgomento e un'ansia smisurati e una solidarietà paterna, - fu quello stato d'estrema lucidità e d'estrema passione a precisare nel mio animo la bontà della missione già intravvista, se una missione avessi dovuto attribuirmi e fossi stato atto a compiere, nelle lettere nostre. (Ungaretti 1974: 743-744)

ciò che grida nell'aria, anche la voce dell'uomo, si metallizza. là.

da questo sotterra si percepisce la melodia delle nostre anime smarrite in un riposo al di

ci guizza uno spasimo felino d'ironie nel sangue che s'agghiaccia, e si modula allontanandosi in un gesto vasto di notte calma» (Ungaretti 1988 : 209). 
Parole simili saranno più volte ripetute in varie occasioni ${ }^{20} \mathrm{e}$ in modo solenne gli capiterà di pronunciarle a Gorizia il 20 maggio 1966 nel cinquantesimo di Porto sepolto. In questo suo discorso Ungaretti insiste ancor di più sul nesso solitudinesofferenza-fraternità (in sostituzione della «solidarietà paterna» del precedente intervento), che arriva - in tempi ormai così diversi - a investire anche il nemico; poiché si tratta di un testo non molto noto ne presentiamo un ampio stralcio, in cui possiamo notare l'insistere sul «luogo nudato» e petroso del Carso come perfetto scenario di un'esperienza di solitudine che le «sventagliature micidiali» muta in urgenza di sentimenti:

Il nome di Gorizia, dopo cinquant'anni torna a significare per me ciò che per noi, soldati in un Carso di terrore, significava allora. Non era il nome di una vittoria - non esistono vittorie sulla terra se non per illusione sacrilega - ma il nome d'una comune sofferenza, la nostra e quella di chi ci stava di fronte e che dicevano il nemico, ma che noi, pure facendo senza viltà il nostro dovere, chiamavamo nel nostro cuore fratello. Ho ripercorso ieri qualche luogo del Carso. Quella pietraia - a quei tempi resa, dalle spalmature appiccicose di fango colore come d'una ruggine del sangue, infida a chi, tra l'incrocio fitto del miagolio delle pallottole, l'attraversava smarrito nella notte - oggi il rigoglio dei fogliami la riveste. È incredibile, oggi il Carso appare quasi ridente. Pensavo: ecco, il Carso non è più un inferno, è il verde della speranza; ecco, pensavo, si fa sede pacifica di poesia, invita a raccolta chi si propone di diffondere poesia, cioè fede ed amore. Ho sbagliato nella mia vita interminabile, tante volte - chi oserebbe contarle, tante sono - e sono difatti un uomo, posso vantarmi di essere stato sempre un uomo anche sbagliando sono un uomo, sono in ogni momento che passa, fallibile; patisco, come ogni altra persona umana, d'abbagli. Ma qui sul Carso, quando mi cavavo dall'anima le parole, le mie povere parole, non sbagliavo. Ero solo, in mezzo ad altri uomini soli. Di null'altro eravamo possessori, noi poveri uomini, se non della propria solitudine, ciascuno. Il luogo era un luogo nudato, un luogo calvo dallo spavento, ma non ne era spaventata la

${ }^{20}$ «Per essere preciso sulla mia vocazione alla poesia, è necessario che ricordi anche questo: che essa mi apparve nettamente durante l'altra guerra. Ero un soldato fra i soldati, vivevo di fronte alla morte, tutte le incrostazioni convenzionali della civiltà mi erano bruscamente sparite di dosso, ero rimasto un povero semplice uomo civile a contatto con la natura che palesava con cruda brutalità il suo prevalere. Da questo attrito è nata la mia poesia. La guerra mi fece capire inoltre che gli uomini, anche i più insigni, anche i più potenti, non erano nulla, ch'essi erano in balia di una sorte che non dipendeva da loro, e che dovevo quindi sentire per essi una solidarietà di fratello. La guerra mi ha insegnato un'altra cosa, a non sprecare le parole. Come non essere laconici, con la minaccia di morte sospesa visibilmente sul vostro capo ad ogni attimo che passa, con il timore di non arrivare in tempo a dire ciò che urgeva dire?... » (Ungaretti 1960: 417).

Nell'intervista di Ferdinando Camon del 1965, «Il Porto Sepolto era la poesia d'un soldato, la poesia d'un uomo esposto alla morte in mezzo alla morte; era magari anche la poesia d'un uomo che accettava con rassegnazione e come una necessità la sofferenza, ma non era certamente un libro che esaltava l'eroismo. Era un libro di compassione del poeta verso di sé, verso i compagni suoi, verso la sorte umana. Era un grido, un'offerta, un'invocazione di fraternità» (Ungaretti 1974: 837). 
nostra anima, era sola, offesa che il nostro corpo fosse, in mezzo a tanta impazienza della morte, tanto, e solo, presente alla propria fragilità. $\mathrm{Fu}$ allora, per in qualche modo guarirci dall'ossessione della fragilità, che nell'anima ci nacque - e crebbe - una forza maggiore e molto più importante della guerra e della morte; fu allora che riudimmo nascere, crescere nell'anima la forza vera, quella che può annientare nell'oblio la solitudine, quella che può muoversi inerme e incolume anche in mezzo al fulmineo, visibile, continuo mietere della morte: era il sentimento ancora tremulo, ancora cauto, ma, come di solito succede alle voci di scoppio primaverile, già, per l'eccesso della delicatezza, troppo impetuoso; era il sentimento che ogni uomo è, senza limitazioni né distinzioni, quando non tradisce se stesso, il fratello di qualsiasi altro uomo, fratello come se l'altro non potesse essergli meno simile d'un altro se stesso. Tornava a nascere tra lo scheggiarsi della roccia in voli di sventagliature micidiali, un sentimento al quale è ancora all'uomo urgente di abilitarsi, finalmente. (Ungaretti 1966: 31-35) ${ }^{21}$

Un'ultima definizione - nelle note autoriali al Meridiano Mondadori del 1969 alza la connotazione drammatica («quella tragedia che portava l'uomo a incontrarsi nel massacro»), ma nello stesso tempo viene ad esaltare il dato di «contraddizione» della vita, già ben espresso dall'antica Veglia ${ }^{22}$.

\section{RIFERIMENTI BIBLIOGRAFICI}

ACCROCCA, Elio Filippo / UNGARETTI, Giuseppe (1960): «Ungaretti, Giuseppe», in Elio Filippo Accrocca (a cura di), Ritratti su misura, Venezia, Sodalizio del libro, pp. 181-183.

BultrinI, Nicola / FABI, Lucio (2007): Pianto di pietra: la Grande Guerra di Giuseppe Ungaretti, Chiari, Nordpress.

CORTEllessa, Andrea (a cura di) (1998): Le notti chiare erano tutte un'alba Antologia dei poeti nella Prima guerra mondiale, Milano, B. Mondadori.

CORTEllessa, Andrea (2000): Ungaretti, Torino, Einaudi.

DE LISO, Daniela (2015): «Di Ungaretti poeta-soldato», Studium, 111, 1, pp. 57-67.

${ }^{21}$ Sull'Ungaretti nel Carso del 1966 vedi la cronaca di Marabini (1973: 135-146).

22 «Nella mia poesia non c'è traccia d'odio per il nemico, né per nessuno: c'è la presa di coscienza della condizione umana, della fraternità degli uomini nella sofferenza, dell'estrema precarietà della loro condizione. C'è volontà d'espressione, necessità d'espressione, c'è l'esaltazione, nel Porto sepolto, quell'esaltazione quasi selvaggia dello slancio vitale, dell'appetito di vivere, che è moltiplicato dalla prossimità e dalla quotidiana frequentazione della morte. Viviamo nella contraddizione... » (Note-Il porto sepolto, 1969, in Ungaretti 2009: 754-55).

Varie le dichiarazioni orali e citate dal vecchio Ungaretti, spesso attestate da Piccioni, tra cui una in equivoca sulla stupidità della guerra («è stata una delle guerre più stupide che si potessero immaginare, a parte che la guerra è sempre stupida; ma quella era particolarmente stupida», in Piccioni 1970 : 60), certo lontana dalle sue mitologie giovanili. 
DE MiCHELIS, Ida (2014): «La grande guerra apocalisse della modernità: Ungaretti e Gadda», Italian Studies in Southern Africa / Studi d'Italianistica nell'Africa Australe, 27 (2), pp. 27-45.

LiVI, François (1988): Ungaretti, Pea e altri. Lettere agli amici egiziani, Napoli, Edizioni scientifiche italiane.

MARABINI, Claudio (1973): La chiave e il cerchio. Ritratti di scrittori contemporanei, Milano, Rusconi, (relativamente al capitolo Con Ungaretti sul San Michele).

PICCIONI, Leone (1970): Vita di Ungaretti, Milano, Rizzoli.

PICCIONI, Leone (1980): Ungarettiana, Firenze, Vallecchi.

SACCONE, Antonio (2012): Ungaretti, Roma, Salerno.

ThOMPSOn, Mark (2009): The White War. Life and Death on the Italian Front, 1915-1919, New York, Basic books.

Ungaretti, Giuseppe (1966): Il Carso non è più un inferno, discorso tenuto a Gorizia 20 maggio 1966, Milano, All'insegna del pesce d'oro.

UngaretTI, Giuseppe (1974): Vita d'un uomo. Saggi e interventi, a cura di Mario Diacono e Luciano Rebay, Milano Mondadori.

UNGARETTI, Giuseppe (1978): Lettere dal fronte a Gherardo Marone (1916-1918), a cura di Armando Marone, introduzione di Leone Piccioni, Milano, Mondadori.

UngaretTi, Giuseppe (1981a): Il Porto sepolto, a cura di Carlo Ossola, Milano, Il Saggiatore.

UngaretTI, Giuseppe (1981b): Lettere a Soffici 1917-1930, a cura di Paola Montefoschi e Leone Piccioni, Firenze, Sansoni.

Ungaretti, Giuseppe (1988) : Lettere a Giovanni Papini 1915-1948, a cura di Maria Antonietta Terzoli, introduzione di Leone Piccioni, Milano, Mondadori.

Ungaretti, Giuseppe (2000): Lettere a Giuseppe Prezzolini 1911-1969, a cura di Maria Antonietta Terzoli, Roma, Edizioni di Storia e Letteratura.

UngaretTI, Giuseppe (2009) : Vita d'un uomo. Tutte le poesie, a cura di Carlo Ossola, Milano, Mondadori.

Ungaretti, Giuseppe (2015): Lettere dal fronte a Mario Puccini, a cura di Francesco De Nicola, Milano, Archinto. 